\title{
Gauthier Bolle, Charles-Gustave Stoskopf, architecte. Les Trente Glorieuses et la réinvention des traditions
}

Rennes, Presses universitaires de Rennes, 2017

\section{Thomas Renard}

\section{(2) OpenEdition \\ Journals}

Édition électronique

URL : http://journals.openedition.org/artefact/1807

DOI : $10.4000 /$ artefact. 1807

ISSN : 2606-9245

Éditeur :

Association Artefact. Techniques histoire et sciences humaines, Presses universitaires du Midi

\section{Édition imprimée}

Date de publication : 30 mai 2018

Pagination : 311-313

ISBN : 978-2-7535-7494-6

ISSN : 2273-0753

\section{Référence électronique}

Thomas Renard, «Gauthier Bolle, Charles-Gustave Stoskopf, architecte. Les Trente Glorieuses et la réinvention des traditions ", Artefact [En ligne], 7 | 2017, mis en ligne le 14 février 2019, consulté le 23 septembre 2020. URL : http://journals.openedition.org/artefact/1807 ; DOI : https://doi.org/10.4000/ artefact. 1807

Ce document a été généré automatiquement le 23 septembre 2020.

\section{cc) (†)}

Artefact, Techniques, histoire et sciences humaines est mise à disposition selon les termes de la Licence Creative Commons Attribution - Pas d'Utilisation Commerciale - Pas de Modification 4.0 International. 


\title{
Gauthier Bolle, Charles-Gustave Stoskopf, architecte. Les Trente Glorieuses et la réinvention des traditions
}

Rennes, Presses universitaires de Rennes, 2017

\author{
Thomas Renard
}

\section{RÉFÉRENCE}

Gauthier Bolle, Charles-Gustave Stoskopf, architecte. Les Trente Glorieuses et la réinvention des traditions, Rennes, Presses universitaires de Rennes, 2017, 356 p.

1 Actif principalement entre l'Alsace et la région parisienne, Charles-Gustave Stoskopf (1907-2004) est l'un des architectes les plus productifs des Trente Glorieuses. À la tête de trois agences ayant réalisé près de 45000 logements, il se présente lui-même comme un des mandarins de l'après-guerre, un architecte qui, à l'image de Bernard Zehrfuss, Jacques Henri Labourdette, Maurice Novarina ou encore Pierre Dufau, a participé à redéfinir par les grands ensembles l'image des villes françaises.

2 Cet ouvrage issu d'une thèse soutenue en 2014 à l'université de Strasbourg sous la direction d'Anne-Marie Châtelet, constitue la première monographie consacrée à cet architecte jusqu'alors relativement peu étudié. Si, comme le reconnait Gauthier Bolle, la volonté initiale était de déterminer le caractère moderne ou régionaliste de l'œuvre de Stoskopf (p. 283), le résultat est nettement plus riche et ambitieux. Au fil de l'analyse, il dresse le portrait d'un architecte charismatique, toujours marqué par l'attachement à la culture de sa région natale et à sa formation académique, et en même temps, tout à fait au fait de la production contemporaine. L'étude formelle et stylistique de sa production n'est qu'une des pistes suivies par l'auteur qui consacre notamment des analyses approfondies aux transformations des techniques de 
construction du logement collectif. De façon plus générale, à travers la figure de Stoskopf, Gauthier Bolle décrit le fonctionnement d'une scène professionnelle de la construction de l'après-guerre, confrontée au besoin impérieux de logement que connait la France.

Sans suivre un strict déroulé chronologique, le plan de l'ouvrage reflète les principaux axes d'analyses choisis par l'auteur. La première partie livre une biographie de l'architecte et dessine les contours de ses réseaux professionnels. Dans la deuxième partie, l'étude de certains chantiers emblématiques permet de dégager trois grandes périodes au sein de l'œuvre de Stoskopf. À partir d'une analyse typo-morphologique, la troisième partie est enfin l'occasion pour $\mathrm{G}$. Bolle de cerner les caractéristiques de la production de l'architecte et de lui donner sa juste place dans l'histoire de l'architecture.

Charles-Gustave Stoskopf grandit et se forme au sein du milieu artistique alsacien, marqué par la figure emblématique de son père, le peintre Gustave Stoskopf. Après un passage à l'École régionale d'architecture de Strasbourg dirigée alors par Robert Danis, il rentre en 1931 à l'École des beaux-arts de Paris où il intègre l'atelier d'Emmanuel Pontremoli remplacé deux ans plus tard par Jacques Debat-Ponsan. Ses succès à l'école sont couronnés en 1933 par l'obtention du deuxième second grand prix de Rome. Dans la capitale, il fréquente également les milieux artistiques, œuvrant notamment durant la guerre comme décorateur pour le cinéma. Si l'Occupation retarde quelque peu les débuts de sa carrière, elle démarre rapidement à la Libération grâce aux liens qu'il sait nouer au sein des milieux institutionnels. Le nouveau ministère de la Reconstruction et de l'Urbanisme le désigne architecte en chef des villages de la « poche » de Colmar. Par la suite, le ministre Eugène Claudius-Petit le nomme architecte-conseil de l'Alsace, position qui le conduit à devenir un véritable mandarin dans sa région natale. Son pouvoir s'étend à l'École régionale de Strasbourg qu'il dirige pendant 18 ans et au sein de laquelle il recrute les meilleurs élèves pour son agence. Il en démissionne en 1967, sceptique quant aux transformations de l'enseignement de l'architecture. Les trois agences qu'il dirige, à Paris, Strasbourg et Colmar, attestent de sa double implantation entre l'Alsace et Paris. L'organisation hiérarchique de ces agences autour du "patron » reflète, à l'image de son enseignement, son attachement au modèle académique.

À partir de 1954, sa collaboration avec la Société Centrale Immobilière de la Caisse des Dépôts et Consignations (SCIC) fait de lui un des acteurs majeurs de la construction des grands ensembles. Nouant des liens d'amitié avec les cadres de la SCIC, il intervient au total dans environ $16 \%$ des logements que la société édifie. L'éloignement de cette société et la mutation de la commande publique entraînent le déclin des agences de Stoskopf à partir des années 1970. G. Bolle tente de dégager les principes qui ont présidé à l'édification des grands ensembles : si Stoskopf souligne lui-même le rôle de contre-modèle qu'ont pu jouer les banlieues pavillonnaires d'avant-guerre, l'adaptation pragmatique aux politiques ministérielles et la recherche d'économie semblent néanmoins prévaloir.

Dans la deuxième partie, Gauthier Bolle opère un choix au sein de l'énorme production de Stoskopf pour analyser les réalisations qu'il estime les plus importantes. Reprenant le déroulement chronologique de sa carrière, chacun des développements est l'occasion d'une remise en contexte plus général, au regard de l'architecture régionaliste (chap. 1), de la reconstruction (notamment à Strasbourg, chap. 2) et de la politique des grands ensembles (chap.3). Consacré à la réinvention du modèle alsacien, le premier 
chapitre traite tour à tour du pavillon alsacien de l'exposition de 1937, où se côtoient des évocations régionales simplifiées et des emprunts à l'Art déco, et la reconstruction d'Ammerschwihr (1945-1949). Approfondissant les analyses de la première partie, Gauthier Bolle voit dans la liberté prise par l'architecte dans cette reconstruction régionale les signes de la réinvention d'un modèle alsacien. De la reconstruction à la question du logement, le deuxième et le troisième chapitre évoquent des chantiers de grande ampleur et notamment le quartier de l'Esplanade de Strasbourg, opération qui occupe l'agence de Stoskopf pendant près de 30 ans et reste selon l'auteur un moment emblématique de l'architecture française des Trente Glorieuses. Cette architecture du grand nombre est étudiée au regard des préceptes académiques enseignés à l'École des Beaux-Arts. Pour chaque cas d'étude, G. Bolle s'attarde plus spécifiquement sur le contexte, la genèse et la réception de l'opération.

7 La dernière partie tente de caractériser à partir de l'analyse typo-morphologique les éléments saillants de l'immense production des agences dirigées par Stoskopf afin de la replacer dans le panorama contemporain de la pratique de l'architecture et de l'urbanisme. L'analyse est particulièrement pertinente quand elle rentre dans le détail des compositions de plans et de l'effort de normalisation, notamment des barres fines, à laquelle Stoskopf participe activement. À l'échelle urbaine, ces travaux révèlent dans les outils de représentation utilisés (importance du plan de masse et des maquettes) et dans ses éléments constitutifs récurrents (axe majeur, place centrale signalée par la verticalité, courbe enserrant un noyau), une persistance de la tradition académique. Stoskopf cherche sans cesse dans ses projets à allier une forme de monumentalité avec une expression épurée. Cette tradition n'exclut pas un regard sur l'architecture contemporaine, notamment du côté de la Scandinavie et des États-Unis (l'auteur cite à plusieurs reprises R. Neutra et M. Breuer), nourri grâce aux voyages d'étude qu'il effectue avec la SCIC. On retrouve l'influence américaine dans certains des équipements qu'il construit (chap. 2), notamment dans les centres commerciaux qui peu à peu prennent une place majeure dans les grands ensembles, tandis que les programmes scolaires prolongent les typologies d'avant-guerre. Programme dominant la tradition académique, les églises occupent une place centrale dans sa production. Ses principales réalisations - notamment l'église de Poissy et le centre diocésain de Créteiltémoignent tout à la fois d'une volonté de monumentalité et d'une recherche de sobriété, voire d'ascèse, que l'auteur met en lien avec ses origines protestantes.

8 Enfin, dans un dernier chapitre regroupant des éléments quelque peu disparates, l'auteur consacre une étude à la technique constructive et à son évolution dans les années 1960 induite par l'emploi de murs porteurs perpendiculaires aux façades et par la préfabrication de celles-ci. L'analyse s'achève par la question de l'héritage alsacien et fait le lien avec le début du récit en évoquant à nouveau son père. L'austérité des grands ensembles n'empêche pas l'auteur de mettre en avant un régionalisme métissé de la production de Stoskopf, soulignant l'adaptation de son parti en fonction des programmes : sinueux, familiers et liés à l'histoire dans les villages et les campagnes alsaciennes; rectilignes et plus rigides dans les banlieues.

En mobilisant de vastes sources archivistiques et orales récoltées lors d'entretiens, Gauthier Bolle réussit à combler un vide historiographique. Au-delà de la simple biographie, l'étude de cet architecte longtemps hégémonique en Alsace permet d'enrichir l'histoire de l'architecture des Trente Glorieuses encore parfois marquée par le paradigme moderniste. À l'heure où plusieurs réalisations de l'architecte ont reçu 
une reconnaissance patrimoniale à travers le label $\mathrm{xx}^{\mathrm{e}}$ siècle (quartier de l'Esplanade et place de l'Homme-de-fer à Strasbourg, reconstruction d'Ammerschwihr, église Saint Louis de Poissy...), l'architecture des logements construits en grand nombre survit mal au passage du temps. L'ouvrage de Gauthier Bolle nous permet de mieux appréhender la question de la reconstruction et surtout des grands ensembles en soulignant tout ce que leur conception doit à la tradition académique.

\section{AUTEURS}

\section{THOMAS RENARD}

Université de Nantes 